\title{
氣 磨の日戀化に就て
}

\section{久保田度，三宅佟三}

On the Diurnal Barometric Oscillations.

By H. R. Kubota and S. MiYake

\begin{abstract}
As the result of examination of Margules' theory on the diurnal barometric oscillations using the observed values of air temperature and atmospheric pressure in the upper air at Lindenberg, we found out that the constancy of the equilibrium vemperature for different altitudes assumed by him is not correct.
\end{abstract}

\section{I，序論}

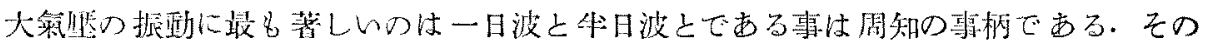
中，坐日波は既に多くの人々に低つて可成渝議されて居る. 然るに一日波はそ孙が主とし

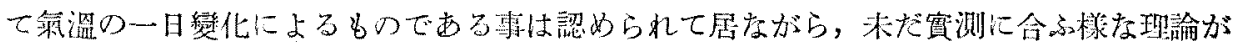

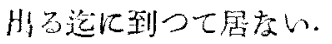

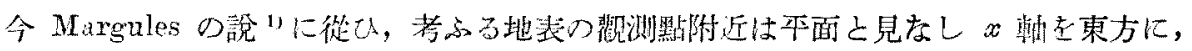

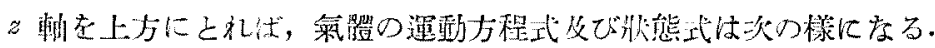

$$
\left.\begin{array}{l}
\frac{\partial u}{\partial t}=-\frac{1}{\rho} \frac{\partial p}{\partial x}: \quad \frac{\partial w}{\partial t}=-\frac{1}{\rho} \frac{\partial p}{\partial z}-g \\
\frac{\partial \rho}{\partial t}+\frac{\partial(\rho u)}{\partial x}+\frac{\partial(\rho w)}{\partial z}=0, \quad p=R \rho T
\end{array}\right\}
$$

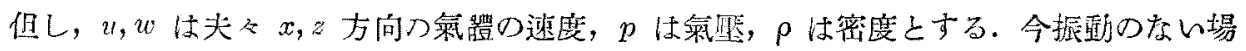

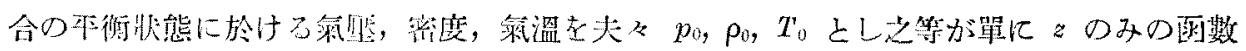
亡すれ代，

$$
\left.\begin{array}{l}
\frac{1}{p_{0}} \frac{d p_{0}}{d z}=-\frac{g}{R T_{1}}, \quad \frac{1}{\rho_{0}} \frac{d \rho_{0}}{d z}=-\frac{g}{R T_{0}}-\frac{1}{T_{0}} \frac{d T_{0}}{d z} \\
p_{0}=R \rho_{i} T_{0}
\end{array}\right\}
$$

$p, \rho$, が振動する時，

$$
p=p_{l}(1+\varepsilon), \rho=\rho_{l}(1+\sigma), T^{\prime}=T_{0}(1+\tau) .
$$

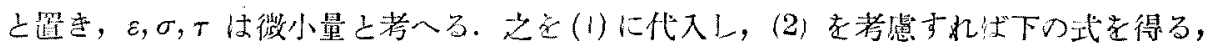

(1) M. Margules: On the Vibration of an Atmosphere Periodically Heated (C. Abbe: The Mechanics of the Earth's Atmosphere, Vol. 2). 


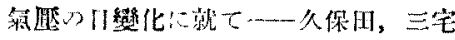

$$
\begin{aligned}
& \frac{\partial^{2} \varepsilon}{\partial x^{2}}+\frac{\partial^{\prime} \varepsilon}{\partial z^{2}}-\frac{g}{R T_{0}^{\prime}} \frac{\partial \varepsilon}{\partial t}-\frac{1}{R T_{0}} \frac{\partial^{2} \varepsilon}{\partial t^{2}} \\
& =\frac{g}{R T_{0}} \frac{\partial \tau}{\partial z}-\frac{g T}{R T_{0}}\left(\frac{g}{R T_{0}}+\frac{1}{T_{0}} \frac{d T_{0}}{d z}\right)-\frac{1}{R T_{0}} \frac{\partial^{2} \tau}{\partial t^{2}}
\end{aligned}
$$

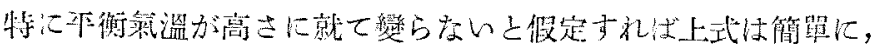

$$
\frac{\partial^{2} \varepsilon}{\partial x^{-1}}+\frac{\partial^{2} \varepsilon}{\partial z^{\prime \prime}}-\alpha \frac{\partial \varepsilon}{\partial z}-\frac{\alpha}{g} \frac{\partial^{?} \varepsilon}{\partial t^{2}}=\alpha \frac{\partial \tau}{\partial z}-\frac{\alpha}{g} \frac{\partial^{2} \tau}{\partial t^{2}}-\alpha^{2} \tau
$$

但L，

$$
\alpha=\frac{g}{R T_{0}}
$$

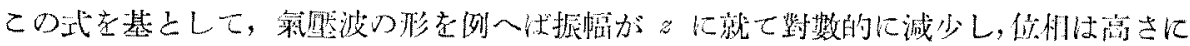

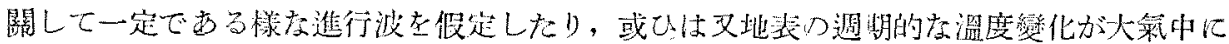

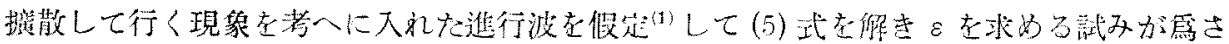

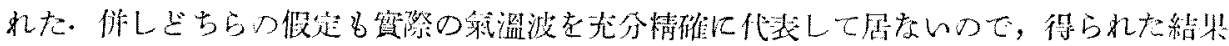
は實测と一政しないわも舆理はない。

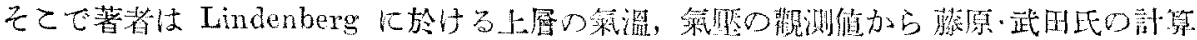
された調和分析の結果

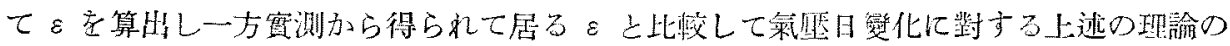
正否考檢する事とした。

\begin{tabular}{|c|c|c|c|c|c|c|}
\hline$Z$ & $\tau_{0}$ & $\tau$ 振幅 & $\tau$ 位相 & $p_{0}$ & $\varepsilon$ 振幅 & $\varepsilon$ 位相 \\
\hline $123 \mathrm{~m}$ & $281.95^{\circ} \mathrm{K}$ & $9.518 \times 10^{-3}$ & $2200^{\circ}$ & $754.38 \mathrm{~mm}$ & $2107 \times 10^{-4}$ & $250^{\circ}$ \\
\hline 500 & 28056 & 3.648 & $186^{1} 1$ & $720 \cdot 52$ & 0416 & 2545 \\
\hline 1005 & 27889 & $1 \cdot 349$ & 1845 & $677 \cdot 95$ & $1 \cdot 106$ & $209 \cdot 3$ \\
\hline 1500 & 275.0 & 1010 & 2115 & 687.52 & 1.850 & 2068 \\
\hline 2000 & 27255 & 0.810 & 2465 & 599.09 & $2 \cdot 403$ & 211.9 \\
\hline 2500 & 3509 & 1237 & 2685 & 56262 & 2773 & 2224 \\
\hline 3000 & 267.28 & 1388 & $266 \cdot 8$ & 527.97 & 3276 & $229 \cdot 7$ \\
\hline 3500 & $264 \cdot 46$ & 1.610 & 2728 & $495 \cdot 19$ & 4059 & 237.8 \\
\hline 4000 & $261 \cdot 55$ & 1.949 & $267 \cdot 1$ & $464 \cdot 13$ & 4977 & 2446 \\
\hline
\end{tabular}

\section{\$II. 計 算の概 要}

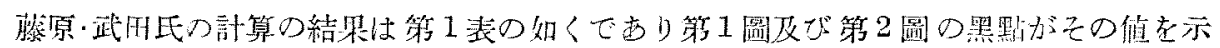
$\frac{1}{9}$.

(1) H. Arakawa: Diurnal Barometric Oscillations as an Effect of a surface Ware cic. Geophys. Mag. Vol. VII, No. 1 (1933).

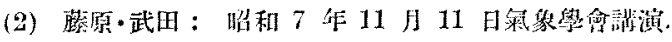




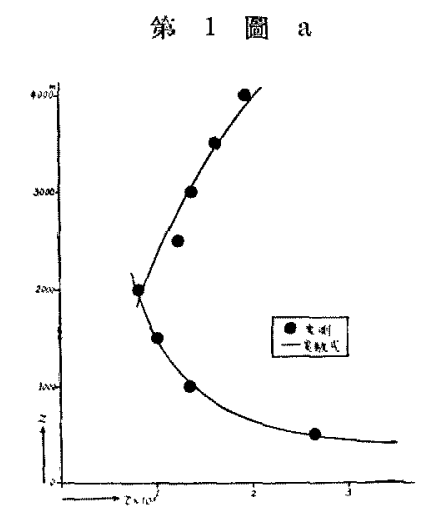

第 1 圆 $b$

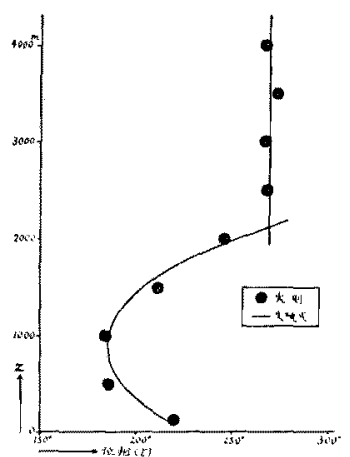

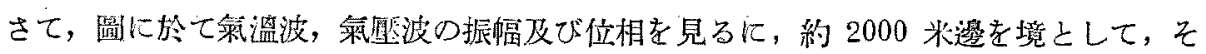

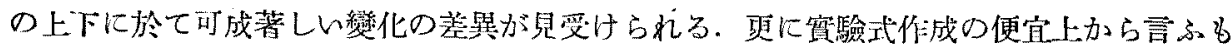

第 2 湎

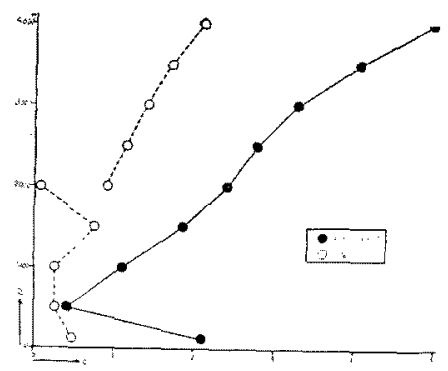

第 2 圖 $b$

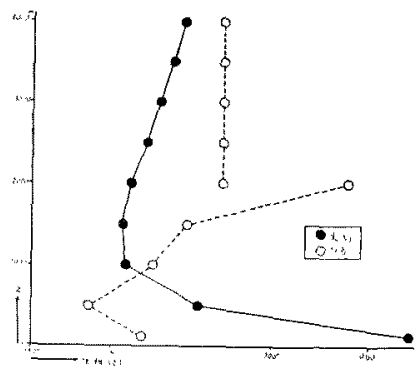

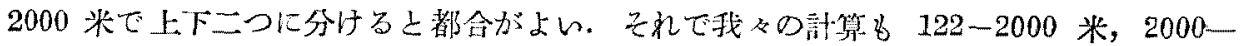

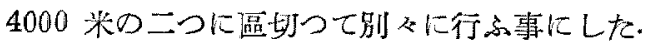

(i) $122-2000$ 米.

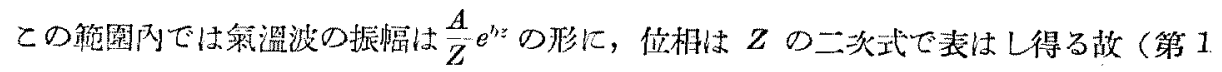
圆䆚照),

$$
\tau=\frac{A}{Z} e^{z z+t\left(2 t+m x+c\left(z-z_{0}\right)^{2}+a\right\}}
$$

と䇫く・となに，

$$
p=\frac{2 \pi}{24 \times 60 \times 60} \text { (地球 }
$$

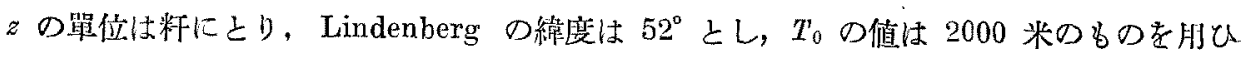
校.

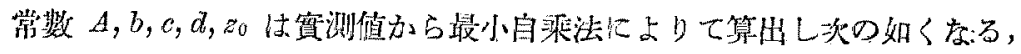




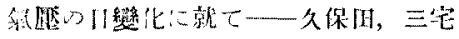

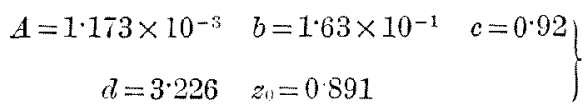

此處で，

$$
\varepsilon=\varepsilon(z) e^{(i t+m x)}
$$

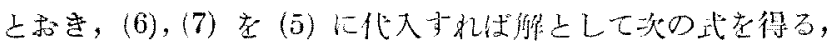

$$
\varepsilon(z)=e^{b z+i z+(\lambda)} \frac{1}{\lambda_{1}-\lambda_{2}}\left\{e^{\lambda_{1}:} \int \chi^{e^{-\lambda_{1} z} d z-e^{\lambda_{2} z}} \int \chi^{z} e^{-\lambda_{2} z} d z\right\} \ldots \ldots \ldots
$$

ていに,

$$
\begin{gathered}
\lambda_{1}, \lambda_{2}=\frac{\alpha}{2}-\left(b-2 i c z_{0}\right) \pm \sqrt{\frac{\alpha^{2}}{4}+m^{2}-\frac{\alpha}{g} p^{9}}, \\
\chi=e^{i c i^{2}}\left\{-\frac{\alpha A}{z^{2}}+\frac{\alpha \Lambda\left(b-i 2 c z_{1}\right)+\frac{p^{2}}{g}-\alpha}{z}+2 i \alpha A c\right\} .
\end{gathered}
$$

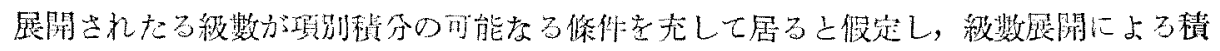

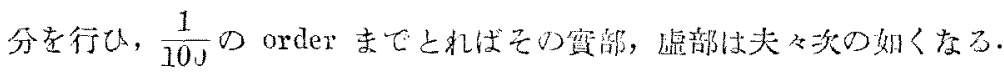

$$
\begin{aligned}
& \mathfrak{H}(\varepsilon(z))=\alpha \cdot 1\left(1+\frac{\alpha}{2} z\right)\left[-\frac{1}{2} c^{2} z_{0}{ }^{4}+z \log z\left\{-\alpha+2\left(c d-c^{2}\right) z_{0}{ }^{3}+\frac{1}{2}(\alpha-b) c^{2} z_{0}^{4}\right\}\right. \\
& +z^{2}\left\{b(b-\alpha)-\left(4 c d-c^{2}\right) z_{0}^{2}+2\left(b c d+b c^{2}-\alpha c^{2}\right) z^{3}\right\} \\
& +z^{3}\left\{\left(c d+c^{2}\right) z_{0}+\left(-2 b c a+\frac{5}{2} b c^{2}+\frac{3}{2} \alpha c^{2}\right) z_{0}^{2}\right\} \\
& \left.+z^{4}\left\{-\frac{1}{2} c^{2}+\left(\frac{2}{3} b c d+2 b c^{2}-\frac{2}{3} \alpha c^{2}\right) z^{\prime \prime}\right\}+z^{5}\left\{-\frac{5}{8} b c^{3}+\frac{1}{8} \alpha c^{2}\right\}\right], \\
& \Im(\varepsilon(z))=\alpha A\left(1+\frac{\alpha}{2} z\right)\left[d z_{0}{ }^{2}+z \log z\left\{2(c-d) z_{0}-\alpha c z_{0}{ }^{2}+c^{2} d z_{0}{ }^{5}\right\}\right. \\
& +2^{2}\left\{c+2(\alpha c-b d) z_{0}+\left(b^{2} c-\alpha b c\right) z_{0}^{2}-\left(4 c^{2} d+c^{3}\right) z_{0}^{2}\right\} \\
& +z^{3}\left\{\left(b c-\frac{1}{2} \alpha c\right)-\left(b^{3} c-\alpha b c\right) z_{11}+\left(3 c^{2} d+2 c^{3}\right) z_{13}^{3}\right\} \\
& \left.+z^{4}\left\{\frac{1}{3} b c(b-\alpha)-2 c^{3} z_{0}-\frac{4}{3} c^{2} d z_{0^{2}}{ }^{2}\right\}+z^{5}\left\{\left(\frac{1}{4} c^{0} d+c^{3}\right) z_{0}\right\}+z^{n}\left\{-\frac{1}{5} c^{3}\right\}\right] .
\end{aligned}
$$

各々の高さに就て，

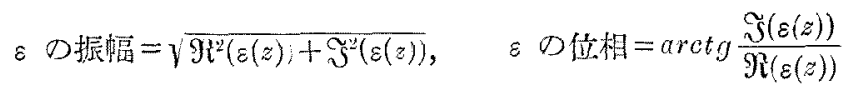

考察際に求めて見名上符 2 表の样になる。 


\begin{tabular}{|c|c|c|c|c|}
\hline & 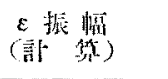 & 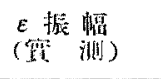 & $\begin{array}{l}\varepsilon \text { 位相 } \\
\text { (棓 䋈) }\end{array}$ & 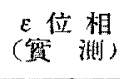 \\
\hline $129 \mathrm{~m}$ & $4673 \times 10^{-6}$ & $2 \cdot 107 \times 10^{-4}$ & $2195^{3}$ & $25.6^{\circ}$ \\
\hline 500 & 2630 & 0.416 & 1864 & $254 \cdot 5$ \\
\hline 1000 & 2493 & $1 \cdot 106$ & $225 \cdot 7$ & 2093 \\
\hline 1500 & $7 \cdot 167$ & 1850 & 247.0 & 2068 \\
\hline 2000 & 0669 & $2 \cdot 403$ & 3466 & 8119 \\
\hline
\end{tabular}

(ii) $2000-4000$ 米.

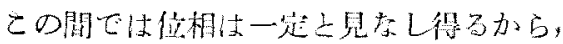

$$
\tau=A^{\prime} e^{3 \prime z+i\left(x+n+n x+e^{\prime}\right)},
$$

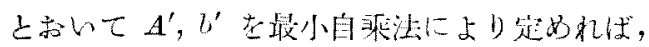

$$
A^{\prime}=4 \cdot 048 \times 10^{-4}, \quad B^{\prime}=4.02 \times 10^{-1} \quad c^{\prime}=269^{\circ}
$$

となる。

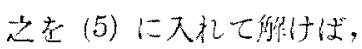

$$
\hat{c}(z)=\frac{a b^{\prime}+\frac{\alpha}{g} p^{2}-\alpha^{2}}{a b^{\prime}-\frac{\alpha}{g} p^{2}-b^{\prime 2}+m^{2}} A^{\prime} c^{b^{\prime} z}
$$

\begin{tabular}{|c|c|c|c|c|}
\hline & 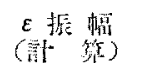 & 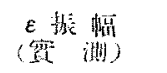 & $\begin{array}{l}\varepsilon \text { 位相 } \\
\text { (部 }\end{array}$ & 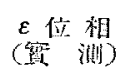 \\
\hline $2000 \mathrm{~m}$ & $090 \times 10^{-3}$ & $2403 \times 10^{-4}$ & $269^{\circ}$ & $2119^{\circ}$ \\
\hline 8500 & $1 \cdot 14$ & 2772 & 259 & 2224 \\
\hline 3000 & 140 & 8.276 & 269 & $229 \cdot 7$ \\
\hline 3500 & $1 \cdot 71$ & 4059 & 289 & $237 \cdot 8$ \\
\hline 4000 & 211 & 4977 & 269 & 2446 \\
\hline
\end{tabular}

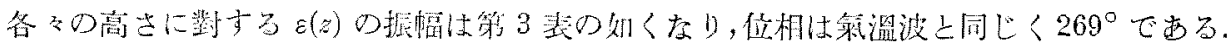

\section{$\S$ III、結論}

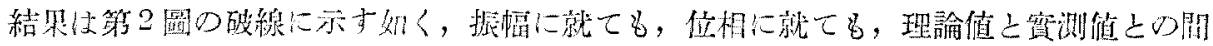

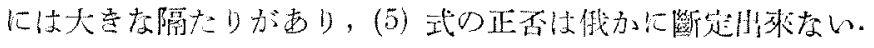

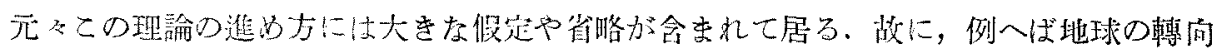

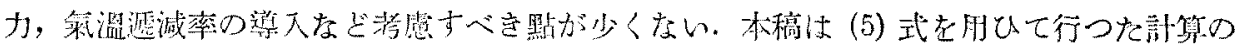

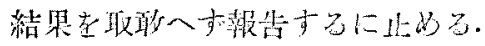




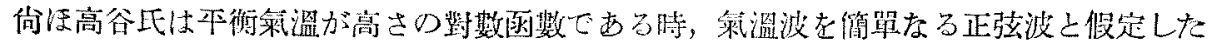

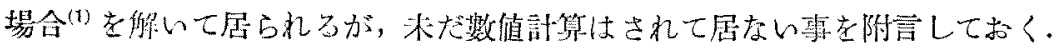

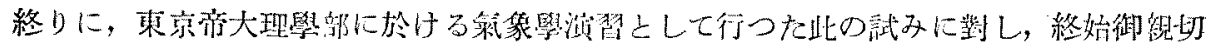

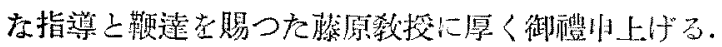

\title{
報 告 Reports
}

\section{大氣壓微振動の觀测結果}

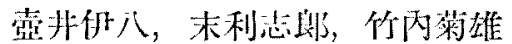

Some Results of the Observation of the Microbarometric Oscillation.

By I. Tubor, S. Suerosi and K. Takeutr.

\begin{abstract}
We andlysed fifty-tbre microbarometric waves (period 5 min.-40 min.) observed at Tokyo in 1933 by the statoscopes and the Yosida's microbarograph at five different places.

Most waves are found to be progressive like three examples reported by Messrs. T. Kita and H. Huzita last year.

In addition, the waves seem to be topographically conditioned, since the direction of their propagation is almost to N, NE or to E., and some waves have local nature. Moreover, their propasating velocities (ca $0.2 \mathrm{~km} / \mathrm{min}$ to ca $4.0 \mathrm{~km} / \mathrm{min}$ ) diminish as they advance.

Daily changes of the wave occurrence are shown in $\$ 5$.
\end{abstract}

\section{\&1. 前 言}

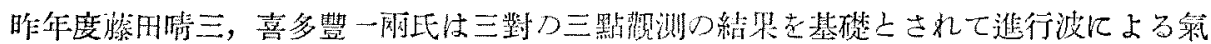

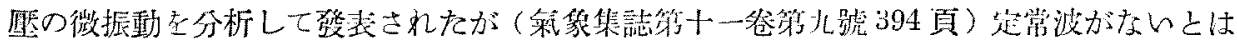

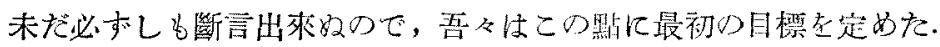

\section{§. 測器及び觀測}

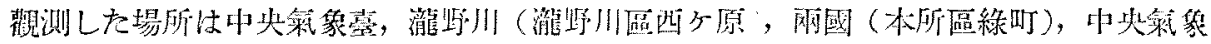

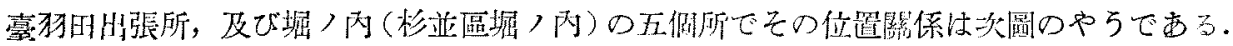

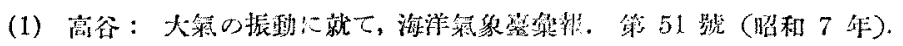

